\title{
Religion et sens de la peine : aux origines théologiques de la peine de prison
}

\section{Éric Wenzel}

\section{(2) OpenEdition}

1 Journals

Édition électronique

URL : https://journals.openedition.org/rdr/1669

DOI : $10.4000 /$ rdr. 1669

ISSN : 2534-7462

Éditeur

Presses universitaires de Strasbourg

\section{Édition imprimée}

Date de publication : 2 décembre 2021

Pagination : 163-173

ISBN : 979-10-344-0097-3

ISSN : 2493-8637

\section{Référence électronique}

Éric Wenzel, «Religion et sens de la peine : aux origines théologiques de la peine de prison », Revue du droit des religions [En ligne], 12 | 2021, mis en ligne le 02 décembre 2021, consulté le 03 mai 2022 URL : http://journals.openedition.org/rdr/1669 ; DOI : https://doi.org/10.4000/rdr.1669

\section{(c) (†) (8)}

La revue du droit des religions est mise à disposition selon les termes de la Creative Commons Attribution - Pas d'Utilisation Commerciale 4.0 International - CC BY-NC 4.0. 


\section{Religion et sens de la peine: aux origines théologiques de la peine de prison}

\section{ÉricWENZEL}

Avignon Université

Université de Montpellier / CNRS, Dynamiques du droit

\section{Résumé}

L'«invention» de la peine de prison par les canonistes médiévaux doit beaucoup à l'adoption par l'Église de la doctrine théologique du libre arbitre, qui induit responsabilité et prise de conscience du condamné, mais également la possibilité d'une réinsertion. La prison moderne, largement fondée sur l'héritage révolutionnaire, qui fait de l'enfermement la clef de voûte du système pénal contemporain, ne se départit pas, à la vérité, de ces fondements religieux. La présence des aumôniers en milieu carcéral n'en trouve que plus de légitimité dans le contexte de la loi de 1905 et de la sécularisation.

\section{Abstract}

The "invention" of the prison sentence by medieval canonists owes much to the adoption by the Church of the theological doctrine of free will, which induces responsibility and awareness on the part of the condemned, but also the possibility of rehabilitation. The modern prison, largely based on the revolutionary legacy, which makes confinement the keystone of the contemporary penal system, does not depart from these religious foundations. The presence of chaplains in prison only finds more legitimacy in the context of the 1905 Act and of secularization. 
$\mathrm{P}$ our bien comprendre le lien entre la ou les religions et la justice pénale, d'une manière générale, et de la peine de prison en particulier, un rappel s'impose sur la dimension religieuse, pendant des millénaires, de la justice. Des «codes» mésopotamiens de la Haute Antiquité à la fin du XVIII ${ }^{\mathrm{e}}$ siècle européen $^{l}$, voire pour certains pays le XIX ${ }^{e}$ siècle inclus, la justice et les éventuelles sanctions en conséquence ont été légitimées par la nature religieuse du pouvoir garant de l'ordre politique et social. Parce que les princes, rois et empereurs tenaient leurs pouvoirs des dieux puis de Dieu, ils étaient légitimes à arbitrer, sanctionner, condamner ou gracier.

Dans ce contexte, les institutions religieuses ont été associées à l'administration de la justice, soit en participant directement à celle-ci (ainsi l'institution ecclésiale invitée par les empereurs romains à assurer un contrôle social sur les populations chrétiennes à la fin de la période antique), soit en accompagnant le bras séculier par des cérémonies destinées à rendre celui-ci plus juste et plus efficace (encore au XVIII ${ }^{\mathrm{e}}$ siècle, avec la pratique de l'amende honorable et la présence de prêtres chargés d'entendre, comme une dernière confession, les paroles du condamné, voire de les faire communier une dernière fois avant de monter sur l'échafaud). Si les anciennes civilisations polythéistes ont eu besoin de s'inventer des dieux ou déesses «spécialisés» dans un idéal de justice (voyez Thémis ou Dikè), c'est que celui-ci nécessitait une hauteur que l'Homme ne possède pas toujours «naturellement». Nos justices contemporaines largement sécularisées portent encore en elles de multiples traces de normes et de pratiques anciennement religieuses; en matière de rituels et de procédures judiciaires, la chose est évidente ${ }^{2}$.

Il convient de rappeler aussi le fait que les trois religions du Livre font doctrinalement de tout comportement déviant un péché devant être puni par les hommes avant de l'être éventuellement par le Créateur; péché qui n'est pas exempt de la rédemption et de pardon de la part des croyants (c'est particulièrement une obligation dans le christianisme) et éventuellement de Dieu («grand miséricordieux»).

1. J. Gaudemet, Les naissances du droit. Le temps, le pouvoir et la science au service du droit, Paris, Montchrestien, 1997 ; F. BLuCHE, «La monarchie absolue de droit divin », in L'Ancien régime: institutions et société, Paris, Fallois, 1993, pour une approche rapide sur la fin de l'Ancien Régime; sur les fondements du droit de punir par les rois absolus, J.-M. CARBASSE, Histoire du droit pénal et de la justice criminelle, Paris, PUF, 2014, p. 141-150.

2. Par commodité, on pourra se référer à É. WenZel (dir.), Justice et religion. Regards croisés: histoire et droit, Avignon, Éd. universitaires d'Avignon, 2008. 
Il s'agit ici de resserrer le lien entre religion et sens de la peine en rapport avec celle de l'enfermement, terme qui a longtemps prévalu sur ceux de prison et d'incarcération.

On rappellera que jusqu'à la fin du XVIII ${ }^{\mathrm{e}}$ siècle, à s'en tenir à l'exemple français, la peine de prison n'existait pas officiellement. Héritage du droit romain (en fait du droit mésopotamien), la prison servait normativement à la prise de corps des accusés; on dirait aujourd'hui à la mise en préventive. La prison ne faisait pas partie de l'arsenal des peines, elle n'était guère prévue par le droit coutumier, même si l'on sait qu'au Moyen Âge les juges seigneuriaux et royaux ont souvent préféré l'alternative de l'enfermement à des glaives plus rédhibitoires ${ }^{3}$. À la vérité, les juges médiévaux n'ont pas initié la peine du cachot, mais ont largement copié un modèle alors légitimé par les clercs.

Là est le cœur du sujet: quel intérêt à la peine de l'enfermement? Pour répondre à cette question, l'historien du droit n'a guère d'autres solutions que de rappeler les fondements religieux et ecclésiastiques de la peine de prison, invention (dans le sens de théorisation) de l'Église médiévale que nos sociétés contemporaines n'ont fait que juridiquement séculariser il y a environ deux siècles, lorsque l'utilitarisme pénal a succédé à la nécessité de la rédemption et du pardon (2).

Or, à l'origine de la peine canonique de prison aux alentours du XII ${ }^{\mathrm{e}}$ siècle, il y a une «révolution» théologique, celle apportée par la scolastique, autour des notions de liberté et de responsabilité (1). La prison est la solution pénale logique à cette orientation spirituelle qui touche alors non seulement le christianisme, mais concomitamment l'islam et le judaïsme, qui semblent pourtant opter plus volontiers pour d'autres modes punitifs ${ }^{4}$.

3. I. Mathieu, "Prisons et prisonniers en Anjou au bas Moyen Âge», Annales de Bretagne et des Pays de l'Ouest, t. 112, 2005.

4. Ainsi en islam, même si le juge religieux peut opter, dans le cadre des peines de correction, pour l'enfermement: non prévu dans le Coran, mais faisant partie de l'arbitraire, au sens juridique du terme, du cadi pour trouver la peine la mieux adaptée. Dans le judaïsme ancien, la peine de prison était inconnue parce que n'appartenant pas aux références vétérotestamentaires. 


\section{LIBRE ARBITRE ET RESPONSABILITÉ : LES FONDEMENTS RELIGIEUX ESSENTIELS DE LA PEINE}

Le libre arbitre est à la théologie ce que la liberté est à la philosophie et au jusnaturalisme. Par opposition au fatalisme et au providentialisme, les tenants du libre arbitre affirment la capacité de l'Homme à agir, ce en conformité avec l'idéal aristotélicien de la raison. Les notions anglaise de Free Will et allemande de Willensfreiheit sont plus éclairantes que le vocable français ${ }^{5}$. Le libre arbitre implique effectivement la volonté parce que l'Homme n'est ou ne serait pas lié aux seuls caprices d'un dieu ou ballotté par les hasards de l'existence.

La notion de libre arbitre est à la vérité antérieure à l'apport de la scolastique médiévale. Saint-Augustin était déjà sans ambiguité sur ce point: «Dieu a conféré à sa créature, avec le libre arbitre, la capacité de mal agir, et par-là même, la responsabilité du péché ${ }^{6}$. Cependant là où le Père de l'Église met en avant le concept pour décharger Dieu de toutes responsabilités vis-à-vis des agissements de sa Créature, la scolastique médiévale en fait un élément fondamental de la Nature humaine, au même titre que la raison, dans un binôme essentiel: la raison est ce qui fonde le libre arbitre.

Thomas d'Aquin, en christianisant la pensée grecque antique (notamment à partir de l'Éthique à Nicomaque d'Aristote), est peut-être celui qui, au Moyen Âge, pousse le plus loin l'existence de la liberté naturelle de l'Homme. En effet, à la différence de Saint-Augustin qui minimise finalement le libre arbitre en matière de salut selon la doctrine de la prédestination, Thomas d'Aquin en fait un élément clef des comportements sociaux et de l'avenir eschatologique du croyant: "L'homme possède le libre arbitre; ou alors les conseils, les exhortations, les préceptes, les interdictions, les récompenses et les châtiments seraient vains ${ }^{7}$ »; ajoutant que la raison est indissociable du libre arbitre. Si tel individu subit des châtiments terrestres et ultérieurement le châtiment divin, c'est parce qu'il a volontairement décidé de mal se conduire. À titre comparatif, le penseur mystique musulman Rûmi, en ce même XIII ${ }^{e}$ siècle, est éclairant de simplicité: on ne donne pas d'ordre à des pierres, parce que la pierre est déraisonnable, au sens premier du terme, donc n'a pas capacité et volonté à agir, en l'occurrence à seule

5. Une approche rapide, mais sérieuse dans J.-B. GulLLon, «Libre arbitre», L'Encyclopédie philosophique, mai 2016: https://encyclo-philo.fr/libre-arbitre-gp [consulté le 28 avr. 2021].

6. Saint-Augustin, Du libre arbitre, I, 16, 35.

7. Somme théologique, I, q. 83, a. 1, rép. 
se déplacer. Un caillou ne peut donc pécher car il n'est pas libre. Déjà au $\mathrm{VII}^{\mathrm{e}}$ siècle, le courant kadarite (qadarite), défendu par l'un des premiers grands savants de l'islam, Hasan Al Basri, puis par les mutazilites, avait fait du libre arbitre l'une des clefs de voûte de la pensée musulmane, faisant écho au Coran et à la forte nuance apportée à la doctrine fataliste: «Dieu ne change pas le destin d'un peuple tant que celui-ci ne change pas ce qui est en lui » (sourate 13, verset 11). Cette position en faveur du libre arbitre et de la raison se retrouve également chez le grand penseur juif Maïmonide au $\mathrm{XII}^{\mathrm{e}}$ siècle: "Par la raison, l'homme distingue le vrai du faux et ceci a lieu dans toutes choses intelligibles ${ }^{8} »$, entendons à l'esprit humain.

Peu importe que le déterminisme ait depuis été énoncé par la sociologie (d'un Bourdieu par exemple) et que la liberté ait été nuancée par la philosophie des sciences (par exemple l'hypothèse d'Everett) et par les neurosciences ${ }^{9}$, on retiendra ici que liberté et responsabilité sont depuis les $\mathrm{XII}^{\mathrm{e}}$-XIII ${ }^{\mathrm{e}}$ siècles au cœur de notre système juridique, au civil comme au pénal. Nos codes contemporains en font foi, au risque d'user d'un mauvais jeu de mots...

Les conséquences en sont notables: le justiciable dont la raison est altérée ne peut être reconnu judiciairement responsable ou pleinement responsable, de même celui que l'on a forcé à commettre une infraction. Le fou, le malade, le domestique que le maître contraint ou le troupeau de moutons qui abîme une parcelle (les Romains avaient déjà tranché la question) ne peuvent donc être tenus responsables par absence ou altération du discernement donc de la volonté. Il en est de même des personnes dont l'âge ou, pendant longtemps, le sexe «faible» est ou a été une justification d'irresponsabilité, totale ou partielle..., ainsi l'énonce la doctrine canonique médiévale.

Surtout, liberté et raison sont deux facteurs déterminants dans la possibilité du rachat et de la rédemption, de la prise de conscience du mal fait, mais aussi du pardon que la société peut accepter d'attribuer à celui qui en a momentanément brisé l'équilibre. À condition que la peine soit éventuellement forte, en fonction de la gravité de la faute, mais non définitive.

Le glissement s'opère alors entre la dimension théologique de la peine et son application par les institutions judiciaires: on comprend dès lors la logique

8. Guide des égarés, $1^{\text {re }}$ partie, chap. 2.

9. P. FABRE, Comprendre le monde pour le changer, Paris, Presses de Sciences Po, 2005, chap. 3 : «L'action individuelle et collective face au déterminisme et à l'imprévisibilité », p. 211-264; M. S. GazZaniga, Le libre-arbitre et les neurosciences, Paris, O. Jacob, 2013. 
de l'invention de la peine de prison par l'institution religieuse, alors même que s'opère l'apport de la scolastique et que l'Église romaine crée ses propres tribunaux, les fameuses officialités puis l'Inquisition chargée de lutter contre ce que l'orthodoxie considère comme des hérétiques. Les $\mathrm{XII}^{\mathrm{e}}$ et $\mathrm{XIII}^{\mathrm{e}}$ siècles sont encore en la matière déterminants, même si un Thomas d'Aquin légitime également la peine de mort, particulièrement contre les criminels impénitents ou d'habitude, comme on appelle alors les récidivistes ${ }^{10}$.

\section{L'« INVENTION » DE LA PEINE DE PRISON : UN HÉRITAGE DE L'ÉGLISE MÉDIÉVALE}

Lorsque le futur conventionnel Lepelletier de Saint-Fargeau, très impliqué dans les débats autour de la réforme judiciaire au début de la Révolution,

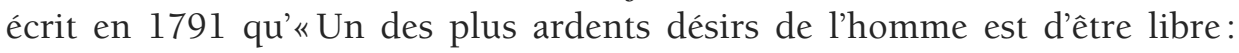
la perte de sa liberté sera le premier caractère de sa peine ${ }^{11}{ }$, il le fait dans un contexte révolutionnaire qui entend faire de la peine de l'enfermement ${ }^{12}$, la clef de voûte du système pénal régénéré ${ }^{13}$. La prison est vue comme la peine et le lieu qui permettent «au condamné de devenir meilleur», donc à être réinséré et à éviter la récidive, obsession des Révolutionnaires puis du XIX ${ }^{\mathrm{e}}$ siècle, comme elle l'était déjà des jurisconsultes au dernier siècle de l'Ancien Régime à la vérité ${ }^{14}$. Nos systèmes judiciaires contemporains sont encore très largement les héritiers de cette philosophie pénale, présentée alors comme résolument moderne. Mais ce futur régicide ne peut méconnaître, en tant qu'ancien procureur général au parlement de Paris, l'apport et l'héritage ecclésiastiques en matière de peine de prison, même si l'époque rejette comme «infame», pour user d'une expression voltairienne, toute influence ecclésiastique dans les normes pénales.

10. C. Gauvard, Condamner à mort au Moyen Âge, Paris, PUF, 2018.

11. Rapport sur le projet du Code pénal, présenté à l'Assemblée nationale, au nom des comités de Constitution et de législation criminelle, par M. Le Pelletier de Saint-Fargeau, présenté le 23 mai 1791: https://www.assemblee-nationale.fr/histoire/peinedemort/rapport_le-pelletierde-saint-fargeau_1791.asp [consulté le 28 avr. 2021].

12. Le vocabulaire juridique de l'époque révolutionnaire use de la peine de l'enfermement plutôt que de celle de la prison.

13. C. CARLIER, «Histoire des prisons et de l'administration pénitentiaire française de l'Ancien Régime à nos jours», Criminocorpus, 14 févr. 2009: http://journals.openedition.org/criminocorpus/246 [consulté le 28 avr. 2021] ; J.-G. PeTIT et al., Histoire des prisons en France, 1789-2000, Toulouse, Privat, 2002.

14. F. BRiegel, É. Wenzel, «La doctrine pénale à l'épreuve de la récidive, 16e siècle-1810», in F. Briegel, M. Porret (dir.), Le criminel endurci. Récidive et récidivistes du Moyen Âge au XXe siècle, Genève, Droz, 2005, p. 93-110. 
Dès le $\mathrm{XII}^{\mathrm{e}}$ siècle, cette peine alors alternative qu'est la prison est pourtant bel et bien légitimée par sa dimension théologique: elle est à l'image du Purgatoire. Ou le condamné prend conscience, grâce à son libre arbitre et à sa raison, du mal qu'il a commis et il pourra être amendé, donc bénéficier d'une réinsertion, ou il entend se maintenir dans une attitude pécheresse et devra passer sa vie cloîtré, comme le pécheur impénitent sera conduit aux Enfers plutôt qu'au Paradis. On passe ainsi symboliquement de l'enfermement (temporaire) à la réclusion (définitive), terme issu du vocabulaire religieux.

Dans ce contexte d'apport de la scolastique: «Les officialités se donnent pour finalité de protéger la société mais aussi de permettre au pêcheur d'obtenir la miséricorde divine» (Denis Salas ${ }^{15}$ ). Comme le pensait déjà le stoïcien Sénèque, aussi remis à l'honneur au Moyen Âge, l'enfermement du corps peut aider à purger l'âme de ses vices, donc à permettre à l'homme de retrouver sa liberté. Entre amendes et excommunication (le bûcher étant une sentence extrême réservée en principe aux hérétiques relaps), la prison ecclésiastique médiévale offre une voie médiane en phase avec une société chrétienne ${ }^{16}$, comme une sorte de pénitence renforcée (au «pain sec et à l'eau» s'ajoutent les murs privatifs de liberté).

Le condamné est alors "cloîtré» autant qu'enfermé car les historiens médiévistes insistent depuis quelques années pour établir un lien entre la vie monacale et la prison. Par similitude antagoniste, le mur des monastères sépare les moines des laiques, la vie présumée exempte de vices et de tentations (héritage du stoïcisme) des péchés de la Cité terrestre, comme le mur des prisons sépare le bon grain social de l'ivraie criminelle. Mais le vocabulaire est édifiant: la cellule du moine est le carcer, qui signifie également prison; mais le cloître (claustrum) renvoie aussi au lieu où l'on enferme les personnes à qui l'Église veut éviter une peine séculière plus lourde (par exemple la mort), comme une protection: une «prison claustrale», à la fois sanction et protection! C'est ainsi qu'environ le tiers des peines canoniques prononcées au Moyen Âge sont des peines d'enfermement, à temps ou (très rarement) définitives, à côté des amendes, des pénitences, des rares excommunications

15. D. SALAS, «Le cloître et la prison. À propos de Enfermements. Le cloître et la prison (VI ${ }^{e}-\mathrm{XVIII}^{e}$ siècles)», Les Cahiers de la justice, 2012/2, p. 187-193; I. Heullant-Donat, J. Claustre, É. Lusset (dir.), Enfermements. Le cloître et la prison (VI ${ }^{e}$-XVIII ${ }^{e}$ siècle), Paris, Publ. de la Sorbonne, 2011.

16. V. Beaulande-BarRaud, «Peines et coercition dans la pratique judiciaire des officialités champenoises (Troyes, Châlons, $\mathrm{XV}^{\mathrm{e}}$ siècle)», in V. Beaulande-Barraud, M. CharaGEAT (dir.), Les officialités dans l'Europe médiévale et moderne: des tribunaux pour une société chrétienne, Turnhout, Brepols, 2014, p. 189-203. 
et du plus exceptionnel bûcher. D'ailleurs un lien évident peut être fait entre pénitence et prison ecclésiastique, nous insistons: en «bon» romaniste ${ }^{17}$ adepte de peines dures, Gratien, dans son célèbre Décret (1140), «milite» pour des cachots sombres dans des lieux coupés du monde afin que le condamné n'ait pas envie de réitérer et préfère participer positivement à son retour dans le corps civique ou la communauté des fidèles, ce qui implique un peu de souffrance christique (théorie démentie par la réalité des prisons médiévales puis de l'Ancien Régime, à la vérité souvent de vraies passoires...). Encore au $\mathrm{XVIII}^{\mathrm{e}}$ siècle, un Dom Mabillon, célèbre historien de la congrégation de SaintMaur, légitime la prison comme un acte de charité (éviter la peine capitale) et de pardon (rachat), là où les philosophes ne pensent qu'en termes d'utilité et d'économie pénale (calcul de la peine juste et paiement de la dette sociale par le travail) : pour finalement se rejoindre sur la réinsertion ${ }^{18}$. Cette réinsertion qui ne disparaît pas complètement après la période des grandes utopies révolutionnaires. Avec les codes napoléoniens, on est encore dans l'idée d'une prison comme «appareil à transformer (positivement) les individus ${ }^{19}$.»

On comprend mieux pourquoi sous la Révolution puis, surtout, au $\mathrm{XIX}^{\mathrm{e}}$ siècle bon nombre d'anciennes abbayes ou d'anciens monastères, comme Clairvaux, Fontevraud ou le Mont-Saint-Michel, vont servir de prison d'État. L'École pénitentiaire des années 1830-1840, qui œuvre sous l'égide d'Arnould Bonneville de Marsangy pour la modernisation du système carcéral (séparation des sexes, des adultes et des enfants, amélioration des cachots insalubres), est un héritage laïcisé de la doctrine canonique médiévale puis moderne tout autant que de la période révolutionnaire ${ }^{20}$. Déjà, entre la fin du Premier Empire et les premières années de la Restauration, des projets réformistes cherchent à aboutir à une prison modèle capable d'assurer privation de liberté, comme idéal de punition, et possibilité de remettre le condamné dans un prétendu droit chemin ${ }^{21}$. Le discours est «laïque»ou «civil», mais assurément nimbé d'une tradition canonique et monastique, en témoigne le recours aux anciens établissements religieux. Dans la maison

17. Nous ne ferons qu'évoquer ici la polémique, déjà ancienne, sur le manque de fiabilité du Décret de Gratien en ce qui concerne ses emprunts au droit romain: la période de la première «redécouverte» du droit romain est en effet propice à certaines réinterprétations voire à «l'invention» de normes présentées comme romaines: A. VeTUlani, "Gratien et le droit romain", Revue historique de droit français et étranger, vol. 24, 1946-1947, p. 11-48.

18. R. Estangüi Gomez, D. Pasquier-Chambolle, «De l'enfermement et des lieux de réclusion », Hypothèses, 2008/1, p. 141-150.

19. M. Foucault, Surveiller et punir. Naissance de la prison, Paris, Gallimard, 1975, p. 269.

20. S. Ruipoli-CAYET, Arnould Bonneville de Marsangy (1802-1894). Un précurseur de la science criminelle moderne, Paris, L'Harmattan, 2002, chap. 2.

21. M. Foucault, op. cit., p. 270-271. 
centrale (1809) puis colonie pénitentiaire de Clairvaux par exemple ${ }^{22}$, dans les anciens bâtiments conventuels, le travail doit accompagner la prise de conscience des détenus aidés par le soutien spirituel d'un personnel ecclésiastique (seize sœurs pour les soins et trois aumôniers), comme naguère il accompagnait la prière collective des moines pour le salut collectif. Les promenades dans l'ancien cloître et les nouveaux quartiers disciplinaires sont censés également permettre de combiner l'idéal d'un triple avantage carcéral: punir, amender, libérer; d'où la création d'une colonie agricole en 1842, réservée aux jeunes asociaux. C'est dans la même veine qu'est également fondée quatre ans plus tard une colonie agricole pénitentiaire pour enfants délinquants dans l'ancienne abbaye de Cîteaux, en Bourgogne. Comme à Clairvaux cependant, les scandales (sanitaires et sexuels) finiront par aller à l'encontre des buts initialement recherchés, entre répression et mansuétude. La prison modèle est une utopie, à laquelle n'échappe pas un Louis-Pierre Baltard (1764-1846) dans son Architectonographie des prisons publiée en 1829 , premier traité d'architecture carcérale ${ }^{23}$. L'auteur n'y cache pas son espérance à œuvrer pour «la guérison des infirmités morales du peuple», à une époque où les classes populaires sont volontiers assimilées à des classes dangereuses. La prison est ici conçue comme un établissement d'éducation et comme un hospice: le projet et le vocabulaire ne sont plus tout à fait religieux, mais ils ne s'en démarquent pas complètement (on y trouve ainsi mention de "discipline intérieure », de «devoir religieux», d' «amendement des coupables», et la proposition de construire des chapelles au centre des nouveaux édifices, etc.). L'ancienne prison Saint-Joseph de Lyon, dans le quartier Perrache, dont la construction débute à la toute fin du règne de Charles X et dont Baltard est le maître d'œuvre, reste imprégnée de l'idée du repentir des condamnés, notamment par la présence de cellules individuelles et par un type architectural qui n'est pas sans rappeler les anciens monastères, notamment par la présence de galeries extérieures proches de la forme des cloîtres (un autre exemple avec, à la même époque, l'ancienne maison d'arrêt de Pontivy en Bretagne avec sa cour centrale destinée à la promenade). La prison voisine de Saint-Paul, construite sous le Second Empire par l'architecte Louvier, montre que l'époque s'engage dans une approche plus fonctionnelle et plus quantitative (aux cellules individuelles de l'édifice précédent répondent des cellules pouvant accueillir plusieurs détenus; des

22. V. le site interactif consacré à Le cloître et la prison. Les lieux de l'enfermement: http:// cloitreprison.fr/ [consulté le 28 avr. 2021].

23. Architectonographie des prisons, ou Parallèle des divers systèmes de distribution dont les prisons sont susceptibles, selon le nombre et la nature de leur population, l'étendue et la forme des terrains, Paris, 1829. 
détenus séparés par quartiers en fonction des types de condamnation) et commence à se détacher davantage encore de l'idéal religieux et ecclésiastique encore bien présent dans la première moitié du siècle. Même si une chapelle centrale y est encore construite, le nouvel édifice carcéral tient davantage du fameux Panoptique - autre utopie d'une prison modèle ${ }^{24}$ - imaginé par Bentham dans les années $1780^{25}$.

Quoi qu'il en soit, encore logique au cœur du système concordataire, le lien entre prison et religion sera néanmoins pour partie conservé par la loi de Séparation de 1905 par le maintien des aumôniers ${ }^{26}$.

En réalité, dès la fin du Moyen Âge et plus encore à partir du Xvie siècle, l'État monarchique, relayé par la doctrine pénale, avait largement contribué dans la pratique à faire de la prison (ou peines assimilées comme les hôpitaux généraux pour les mendiants et prostituées, voire la Bastille pour les affaires politiques) une alternative aux peines «légales» infamantes et afflictives (mort, bannissement, galères ou bagnes ${ }^{27}$ ), dans un «savant» mélange entre économie pénale et utopie de l'enfermement salvateur. On ne sera donc pas étonné de la fondation de l'aumônerie générale des galères au Grand Siècle et dont le célèbre Saint-Vincent de Paul a été le premier titulaire.

De la prison ecclésiastique à la prison d'État, du libre arbitre à la responsabilité codifiée, la généalogie historique et juridique est assurément des plus flagrantes. Aujourd'hui, si les officialités ne condamnent plus à la prison

24. Une approche synthétique de la pensée benthamienne dans M. SCHMID, «La mascarade des coupables: le jeu des masques dans Le Panoptique de Bentham», Laval théologique et philosophique, vol. 60, no 3, 2004, p. 543-555: https://id.erudit.org/iderudit/011364ar [consulté le 3 mai 2021].

25. Que Monsieur Frédéric Didier, architecte en chef des monuments historiques, soit ici remercié pour ces précisions sur ces deux prisons lyonnaises. Est-ce entièrement un hasard si ces deux bâtiments ont été reconvertis en campus de l'Université catholique de Lyon? L. Bunel, "Vue et historique des prisons de Perrache», Criminocorpus, 2011, mis à jour en 2014: http://patrimoinecarceral.blogspot.com/2011/04/vue-et-historiquedes-prisons-de.html [consulté le 3 mai 2021].

26. M. Clavier, «La prison, "laboratoire" d'Église», Nouvelle revue de théologie, 2004/3, p. 398-411: https://www.cairn.info/revue-nouvelle-revue-theologique-2004-3-page-398. htm [consulté le 3 mai 2021]; art. 2 de la loi de 1905: "Pourront toutefois être inscrites auxdits budgets [de l'État] les dépenses relatives à des services d'aumônerie et destinées à assurer le libre exercice des cultes dans les établissements publics tels que lycées, collèges, écoles, hospices, asiles et prisons.»

27. Le bagne, qui remplace officiellement les galères en 1748, cumule idéal de l'enfermement et économie pénale, mais il s'insère davantage dans la réclusion par les longues peines qui l'accompagnent et par la difficulté de la réinsertion du fait de la marque (le G sur l'épaule) apposée sur les corps des condamnés. 
depuis longtemps, se réservant la discipline professionnelle ecclésiastique et matrimoniale, les Églises chrétiennes n'ont pas pour autant déserté le monde des prisons qu'elles ont largement contribué à initier. L'idée de la rédemption du pécheur est encore d'actualité, faisant écho aux propos, assurément partagés dans leur fondement par les instances protestantes voire celles représentatives d'autres cultes reconnus, d'un Père Lataste (1581-1660), le fondateur des Sœurs dominicaines de Béthanie, très engagées dans l'apostolat en milieu carcéral: «Il est donc vrai que les plus grands pécheurs ont en eux ce qui fait les plus grands saints. Qui sait s'ils ne le deviendront pas un jour? » Conservons un brin d'utopie, sous peine de ne retenir de nos centres pénitentiaires que leurs propensions criminogènes... 